\title{
Families of magnetic semiconductors - an overview
}

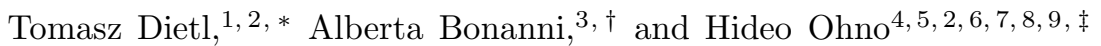 \\ ${ }^{1}$ International Research Centre MagTop, Institute of Physics, \\ Polish Academy of Sciences, Aleja Lotnikow 32/46, PL-02668 Warsaw, Poland \\ ${ }^{2}$ WPI Advanced Institute for Materials Research, Tohoku University, \\ 2-1-1 Katahira, Aoba-ku, Sendai 980-8577, Japan \\ ${ }^{3}$ Institut für Halbleiter- und Festkörperphysik, Johannes Kepler University, Altenbergerstrasse 69, A-4040 Linz, Austria \\ ${ }^{4}$ Laboratory for Nanoelectronics and Spintronics, \\ Research Institute of Electrical Communication, Tohoku University, \\ 2-1-1 Katahira, Aoba-ku, Sendai 980-8577, Japan \\ ${ }^{5}$ Center for Science and Innovation in Spintronics (Core Research Cluster) \\ Tohoku University 2-1-1 Katahira, Aoba-ku, Sendai 980-8577, Japan \\ ${ }^{6}$ Center for Spintronics Integrated System, Tohoku University, \\ 2-1-1 Katahira, Aoba-ku, Sendai 980-8577, Japan \\ ${ }^{7}$ Center for Innovative Integrated Electronic Systems, Tohoku University, \\ 468-1 Aramaki Aza Aoba, Aoba-ku, Sendai 980-0845, Japan \\ ${ }^{8}$ Center for Science and Innovation in Spintronics (Core Research Cluster), \\ Tohoku University, 2-1-1 Katahira, Aoba-ku, Sendai 980-8577, Japan \\ ${ }^{9}$ Center for Spintronics Research Network, Tohoku University, \\ 2-1-1 Katahira, Aoba-ku, Sendai 980-8577, Japan
}

\begin{abstract}
The interplay of magnetic and semiconducting properties has been in the focus since more than a half of the century. In this introductory article we briefly review the key properties and functionalities of various magnetic semiconductor families, including europium chalcogenides, chromium spinels, dilute magnetic semiconductors, dilute ferromagnetic semiconductors and insulators, mentioning also sources of non-uniformities in the magnetization distribution, accounting for an apparent high Curie temperature ferromagnetism in many systems. Our survey is carried out from today's perspective of ferromagnetic and antiferromagnetic spintronics as well as of the emerging fields of magnetic topological materials and atomically thin 2D layers.
\end{abstract}

Key words: Magnetic and dilute magnetic semiconductors; topological materials; 2D systems

DOI: $\quad$ PACC: 7550 P

\section{Magnetic semiconductors}

The discovery of ferromagnetism in some europium chalcogenides and chromium spinels a half of the century ago ${ }^{1]}$ came as a surprise, since insulators typically showed antiferromagnetic or ferrimagnetic spin ordering, driven by a superexchange interaction, whereas ferromagnetism was considered a domain of metals. However, the Goodenough-Kanamori-Anderson rules indicate in which cases the superexchange can lead to ferromagnetic shortrange coupling between localized spins. This mechanism accounts e.g. for Curie temperature $T_{\mathrm{C}}=130 \mathrm{~K}$ in $\mathrm{CdCr}_{2} \mathrm{Se}_{4}{ }_{2]}^{[2]}$. In the case of $\mathrm{EuO}$ and EuS, the antiferromagnetic superexchange is overcompensated by a direct $f-d$ ferromagnetic exchange, which results in $T_{\mathrm{C}}=68 \mathrm{~K}$ and $16 \mathrm{~K}$, respectively $[3]$.

Soon after their discovery, magnetic semiconductors were found to exhibit outstanding properties, including

\footnotetext{
*dietl@ifpan.edu.pl

$\dagger$ alberta.bonanni@jku.at

$\ddagger$ ohno@riec.tohoku.ac.jp
}

colossal magnetoresistance and magnetooptical effects, assigned rightly to the exchange interaction of band carriers and localized spins. This $s d-f$ coupling results in a giant spin splitting of bands below $T_{\mathrm{C}}$, as well as in magnetization fluctuations near $T_{\mathrm{C}}$ generated by band carriers $\left.{ }^{4}, 5\right]$. Due to the presence of a sizable $s d-f$ exchange interaction, by electron doping (oxygen vacancies, Eu substituted by Gd) the magnitude of $T_{\mathrm{C}}$ can be enhanced by about $50 \mathrm{~K}$ in $\mathrm{EuO}$, in agreement with the Ruderman-Kittel-Kasuya-Yosida (RKKY) theory ${ }^{3}$.

More recently, EuS and related ferromagnetic insulators have been proposed as functional overlayers that can lead to novel topological properties by exchange splitting of interfacial bands via a ferromagnetic proximity effect ${ }^{[6]}$. At the same time, $\mathrm{HgCr}_{2} \mathrm{Se}_{4}\left(T_{\mathrm{C}}=110 \mathrm{~K}\right)$ appears to be a Weyl semimetal ${ }^{7}$. Furthermore, antiferromagnetic EuTe constitutes a test-bench for the emerging field of antiferromagnetic spintronics ${ }^{[8]}$.

Another class of materials that attract considerable attention are van der Waals magnetic semiconductors studied down to the atomically thin limit ${ }^{[9]}$. A competition between ferromagnetic and antiferromagnetic superexchange accounts for the magnetic properties of $\mathrm{CrI}_{3}$ and related systems as a function of the number of layers, electric field, and strain[9, 10]. In particular, monolayers are ferromagnetic in the case of these compounds in which, similarly to $2 \mathrm{D}(\mathrm{Cd}, \mathrm{Mn}) \mathrm{Te}$ [11] , the lowtemperature $2 \mathrm{D}$ spin order is stabilized by the large magnitude of the uniaxial magnetic anisotropy ${ }^{12}$. On the 
other hand, $\mathrm{TMPX}_{3}$, where TM is a transition metal and $\mathrm{X}$ is either $\mathrm{S}$ or Se, are antiferromagnetic semiconductors. Their properties as a function of the layer number are under investigations 9 .

\section{Dilute magnetic semiconductors}

This material family was initially named semimagnetic semiconductors ${ }^{[13]}$, as it comprises standard semiconductors doped with magnetic impurities that are randomly distributed, electrically inactive, and do not show any long-range spatial spin ordering 14, 15]. Typical representatives are $(\mathrm{Cd}, \mathrm{Mn}) \mathrm{Te},(\mathrm{Zn}, \mathrm{Co}) \mathrm{O}$, and $(\mathrm{Pb}, \mathrm{Eu}) \mathrm{S}$. Here, the short-range antiferromagnetic superexchange between $\mathrm{Mn}$ [16, 17] and heavier $3 d$ transition metal ions (e.g. Co) or long-range dipolar interactions between rare earth magnetic moments ${ }^{18}$ lead to spin-glass freezing at $T_{\mathrm{f}}<1 \mathrm{~K}$ for $x<0.1$. Magnetooptical and quantum transport experiments have allowed to reveal and quantify the influence of $s p-d$ coupling upon the exciton, polariton, and Landau level spectra, quantum Hall effects, one-electron and many-body quantum localization, universal conductance fluctuations, and carrier spin dynamics in dilute magnetic semiconductor systems of various dimensionalities realized in bulk, $2 \mathrm{D}$ quantum structures, nanowires, and quantum dots $[15,19 \sqrt[19]{21}$, as well as made it possible to optically detect electrical spin injection 22]. Conversely, the dynamics of localized spins has been probed by the Faraday effect 23$]$ or quantum noise spectroscopy [24]. The physics of bound magnetic polarons, single electrons interacting with spins localized within the confining potential of shallow impurities or quantum dots, has been advanced in dilute magnetic semiconductors 25$]$. While the majority of experimental results can be explained within virtual crystal and molecular field approximations, strong coupling effects show up in the case of oxides and nitrides doped with transition metal impurities 26, 27].

Most of the end binary compounds, for instance MnSe and $\mathrm{EuTe}$, are insulating antiferromagnets and, therefore, attract attention from the point of view of antiferromagnetic spintronics. Another ultimate limit of dilute magnetic semiconductors is represented by qubit systems consisting of single magnetic ions in single quantum $\operatorname{dots}[28,29]$.

\section{p-type dilute ferromagnetic semiconductors}

In these systems, a high density of delocalized or weakly localized holes leads to long-range ferromagnetic interactions between transition metal cations, which dominate over the antiferromagnetic superexchange, and are well described by the $p$-d Zener model [30, 31], more universal compared to RKKY-type of approaches. The flagship example of this material family is $(\mathrm{Ga}, \mathrm{Mn}) \mathrm{As}$ in which Mn ions introduce spins and holes to the valence band ${ }^{[32]}$, but to this category belong also other types of magnetically doped p-type compounds, in which holes originate from point defects, like $(\mathrm{Pb}, \mathrm{Sn}, \mathrm{Mn}) \mathrm{Te}$ [33] , or from shallow acceptor impurities, e.g., $(\mathrm{Cd}, \mathrm{Mn}) \mathrm{Te} /(\mathrm{Cd}, \mathrm{Mg}) \mathrm{Te}: \mathrm{N}^{11]}$ and $(\mathrm{Zn}, \mathrm{Mn}) \mathrm{Te}: \mathrm{N}^{[34]}$, rather than from $\mathrm{Mn}$ ions. The reported magnitudes of Curie temperature $T_{\mathrm{C}}$ reach $200 \mathrm{~K}$ in $(\mathrm{Ga}, \mathrm{Mn}) \mathrm{As}^{[35-37]}$, $\left.(\mathrm{Ge}, \mathrm{Mn}) \mathrm{Te}{ }^{[38,}, 39\right]$, and $(\mathrm{K}, \mathrm{Ba})(\mathrm{Zn}, \mathrm{Mn})_{2} \mathrm{As}_{2}[40]$ with less than $10 \%$ of Mn cations, $x_{\text {eff }}<0.1$, measured by saturation magnetization in moderate fields, $\mu_{0} H \lesssim 5 \mathrm{~T}$. The values of Curie temperatures achieved in p-type dilute ferromagnetic semiconductors are to be contrasted with $T_{\mathrm{C}} \approx 0.16 \mathrm{~K}$ in $\mathrm{n}-\mathrm{Zn}_{1-x} \mathrm{Mn}_{x} \mathrm{O}$ :Al with $x=0.03^{41]}$. Such low values of $T_{\mathrm{C}}$ in n-type systems reflect the relatively small magnitudes of both $s-d$ exchange integral and density of states. Higher $T_{\mathrm{C}}$ values are observed only in specific situations, for instance, at the crossings of electron Landau levels under quantum Hall effect conditions ${ }^{42]}$.

Ground breaking spintronic functionalities have been demonstrated and theoretically described for $(\mathrm{Ga}, \mathrm{Mn}) \mathrm{As}$ and related systems 31,43$]$. They rely on the strong $p$ $d$ coupling between localized spins and hole carriers, as well as on sizable spin-orbit interactions in $p$-like orbitals forming the valence band or originating from inversion asymmetry of the host crystal structure. Numerous functionalities of $(\mathrm{Ga}, \mathrm{Mn}) \mathrm{As}$ and other p-type dilute ferromagnetic semiconductors (electrical spin injection ${ }^{44]}$, magnetization control by an electric field $45-47]$, currentinduced domain-wall motion [48, 49], anisotropic tunneling magnetoresistance 50, 51], and spin-orbit torque ${ }^{[52,[53]}$ ) have triggered the spread of spintronic research over virtually all materials families. In particular, they are now explored in multilayers of transition metals supporting ferromagnetism above room temperature ${ }^{[54}$. At the same time, the search for high $T_{\mathrm{C}}$ ferromagnetic semiconductors led to the discovery of Fe-based superconductors, whereas theoretical approaches to the anomalous Hall effect in $(\mathrm{Ga}, \mathrm{Mn})$ As in terms of the Berry phase ${ }^{[55, \text {, [56] }}$ and studies of spin-orbit torque in the same compound [52, 53] have paved the way to spintronics of topological [57, 58] and antiferromagnetic systems ${ }^{[59]}$.

\section{Dilute ferromagnetic insulators}

As mentioned, the Goodenough-Kanamori-Anderson rules indicates in which cases superexchange can lead to ferromagnetic short-range coupling between localized spins. According to experimental and theoretical studies, such a mechanism operates for $\mathrm{Mn}^{3+}$ ions in $\mathrm{GaN}$ and accounts for $T_{\mathrm{C}}$ values reaching about $13 \mathrm{~K}$ at $x \approx 10 \%$ in semi-insulating wurtzite $\mathrm{Ga}_{1-x} \mathrm{Mn}_{x} \mathrm{~N}$ [60], an accomplishment preceded by a long series of material development stages 61, 62]. Since there is no competing antiferromagnetic interactions and due to high atom density, an unusually high magnitude of magnetization for the dilution $x=0.1$ is observed in $\mathrm{Ga}_{1-x} \mathrm{Mn}_{x} \mathrm{~N}^{62]}$. In contrast to random antiferromagnets (such as II-VI dilute mag- 
netic semiconductors), there is no frustration in the case of ferromagnetic spin-spin interactions. Furthermore, according to the tight-binding theory, the interaction energy decays exponentially with the spin-spin distance $[60]$. Accordingly, the dependence of $T_{\mathrm{C}}$ on $x$ cannot be explained within the mean-filed approximation, but corroborates the percolation theory ${ }^{63}$, and can be quantitatively described by combining tight-binding theory and Monte Carlo simulations [60].

The significant inversion symmetry breaking specific to wurtzite semiconductors and the insulating character of the system allow controlling the magnetization by an electric field via a piezoelectromagnetic coupling in $(\mathrm{Ga}, \mathrm{Mn}) \mathrm{N}$. As shown experimentally and confirmed theoretically [64], the inverse piezoelectric effect changes the magnitude of the single-ion magnetic anisotropy specific to $\mathrm{Mn}^{3+}$ ions in GaN, and thus the magnitude of magnetization.

\section{Dilute magnetic topological materials}

In these systems $[57,[58]$, there are novel consequences of magnetization-induced giant $p-d$ exchange spin-splittings of bulk and topological boundary states. In particular, in thin layers of $3 \mathrm{D}$ topological insulators, this splitting turns hybridized topological surface states into chiral edge states. Striking consequences of this transformation include: (i) the precise quantization of the Hall conductance $\sigma_{x y}=e^{2} / h$ demonstrated for thin layers of ferromagnetic $(\mathrm{Bi}, \mathrm{Sb}, \mathrm{Cr})_{2} \mathrm{Te}_{3}$ at millikelvin temperatures ${ }^{65]}$, as predicted theoretically ${ }^{66]}$; (ii) the efficient magnetization switching by spin-locked electric currents in these ferromagnets 67 . In the case of 3D Dirac materials with magnetic impurities coupled by antiferromagnetic superexchange, such as $(\mathrm{Cd}, \mathrm{Mn})_{3} \mathrm{As}_{2}$ or strained $(\mathrm{Hg}, \mathrm{Mn}) \mathrm{Te}$, the magnetic field-induced formation of Weyl semimetals with non-zero topological charges has been predicted [68].

Furthermore, inverted band ordering specific to topological matter enhances the role of the long-range interband Bloembergen-Rowland contribution to spin-spin interactions, resulting in higher spin-glass freezing temperatures $T_{\mathrm{f}}$ in topological semimetals, such as $(\mathrm{Cd}, \mathrm{Mn})_{3} \mathrm{As}_{2}$ and $(\mathrm{Hg}, \mathrm{Mn}) \mathrm{Te}$, compared to topologically trivial IIVI Mn-based dilute magnetic semiconductors 17 . This spin-spin exchange mechanism, taken into consideration within the $p-d$ Zener model ${ }^{699}$ and named Van Vleck's paramagnetism, was proposed to lead to ferromagnetism in topological insulators of bismuth/antimony chalcogenides containing $\mathrm{V}, \mathrm{Cr}$, or $\mathrm{Fe}^{[66]}$, a proposal discussed earlier in the context of zero-gap ( $\mathrm{Hg}, \mathrm{Mn}) \mathrm{Te}$ 70]. However, according to $a b$ initio studies and corresponding experimental data, the superexchange in the case of $\mathrm{V}$ and $\mathrm{Cr}$, and the RKKY coupling in Mn-doped films appear to contribute significantly to ferromagnetism in

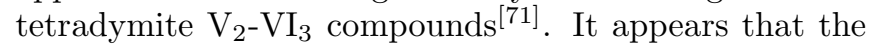
co-existence of ferromagnetic superexchange with ferro- magnetic Bloembergen-Rowland and RKKY interactions accounts for $T_{\mathrm{C}}$ as high as $250 \mathrm{~K}$ in $\left(\mathrm{Cr}_{x} \mathrm{Sb}_{1-x}\right)_{2} \mathrm{Te}_{3}$ with $x=0.44^{\text {[2] }}$. Ferromagnetic coupling between magnetic impurities mediated by carriers residing in surface or edge Dirac cones is rather weak for realistic values of spatial extension of these states into the bulk ${ }^{[31]}$.

\section{Heterogeneities in magnetic semiconductors}

It becomes increasingly clear that spatial inhomogeneities in the magnetization distribution account for a number of key properties of magnetic semiconductors, including the magnitude of $T_{\mathrm{C}}$. We discuss here three distinct mechanisms accounting for the non-uniform distribution of magnetization and describe their consequences.

(1) According to the percolation theory applicable to dilute systems with short-range spin-spin interactions, critical temperature $T_{c}$ corresponds to the formation of a percolative cluster that incorporates typically about $16 \%$ of the spins in the 3D case. This cluster extends over all spins at $T=0$, implying that at $T>0$ ferromagnetism co-exists with superparamagnetism produced by not percolative clusters $[$ 73]. Presumably this effect accounts for thermal instabilities of the quantum anomalous Hall effect even at $T \ll T_{\mathrm{C}}$, and shifts below $100 \mathrm{mK}$ the operation range of the potential resistance standards based on this phenomenon 74,75$]$.

(2) A specific feature of conducting magnetic semiconductors is the interplay of carrier-mediated ferromagnetism with carrier localization, which results in spatial fluctuations of magnetization and superparamagnetic signatures generated by the critical fluctuations in the carrier density of states in the vicinity of the metal-toinsulator transition. This effect has been extensively discussed in the context of both ferromagnetic ${ }^{[5,}$, 76] and dilute ferromagnetic semiconductors $\frac{\text { 77 } 79] \text {. }}{\text { 7 }}$.

(3) The magnetic properties discussed above have concerned systems with a random distribution of cationsubstitutional magnetic impurities. A major challenge in dilute magnetic materials is the crucial dependence of their properties on the spatial distribution of magnetic ions and their position in the crystal lattice. These nanoscale structural characteristics depend, in turn, on the growth and processing protocols, as well as on doping with shallow impurities [80, 81]. By employing a range of photon, electron, and particle beam methods, with structural, chemical, and spin resolution down to the nanoscale, it has become possible to correlate the surprising magnetic properties with the spatial arrangement and with the electronic configuration of the magnetic constituent [82, [83]. In particular, the aggregation of magnetic cations which are either introduced deliberately or present due to contamination, accounts for the high $T_{\mathrm{C}}$ values observed in a number of semiconductors and oxides 84, 85]. The symmetry lowering associated with this aggregation is also responsible for remarkable magnetic and magnetotransport anisotropy properties of 
$(\mathrm{Ga}, \mathrm{Mn}) \mathrm{As}^{[86]}$ and $(\mathrm{In}, \mathrm{Fe}) \mathrm{As}^{87]}$.

\section{Outlook}

Semiconductors and insulators doped with magnetic impurities, in addition to showing fascinating physics, have well established applications as semi-insulating substrates and layers, and as building-blocks for solid state lasers, optical insulators, and detectors of high energy photons. More recent works have revealed surprising materials science features, such as a dependence of the spatial distribution of magnetic impurities on the Fermi level position. At the same time, studies of dilute ferromagnetic semiconductors have led to discoveries of breakthrough functionalities, like for instance spin-orbit torque and magnetization manipulation by an electric field, which are on the way of recasting the concept of computer hardware. The rise of magnetic quantum materials opens for magnetic semiconductors a new chap- ter involving magnetic proximity effects, functionalities of topological boundary states, and the control of van der Waals heterostructures. It is justified to expect that research in semiconductors, insulators, and organic materials containing magnetic constituents, and particularly in their multilayers and quantum structures, will continue to generate unanticipated and inspiring discoveries in the years to come.

\section{ACKNOWLEDGEMENTS}

The work of T.D. is supported by the Foundation for Polish Science through the IRA Programme financed by EU within SG OP Programme. A. B. acknowledges a support by the Austrian Science Foundation - FWF (P31423 and P26830) and by the Austrian Exchange Service (ÖAD) project PL-01/2017.
[1] S. Methfessel and D.C. Mattis, in Magnetic Semiconductors, Handbuch der Physik, Vol. 18, edited by S. Flugge (Springer, 1968) p. Pt. 1.

[2] P. K. Baltzer, P. J. Wojtowicz, M. Robbins, and E. Lopatin, "Exchange interactions in ferromagnetic chromium chalcogenide spinels," Phys. Rev. 151, 367-377 (1966).

[3] P. Wachter, "Europium chalcogenides: EuO, EuS, EuSe and EuTe," in Handbook on the Physics and Chemistry of Rare Earth, Vol. 2, edited by Jr. K. A. Gschneidner and L. Eyring (North-Holland, Amsterdam, 1979) p. 507.

[4] T. Kasuya and A. Yanase, "Anomalous transport phenomena in Eu-chalcogenide alloys," Rev. Mod. Phys. 40, 684-696 (1968)

[5] E. L. Nagaev, "Colossal-magnetoresistance materials: manganites and conventional ferromagnetic semiconductors," Phys. Rep. 346, 387 (2001)

[6] I. Žtić, A. Matos-Abiague, B. Scharf, H. Dery, and K. Belashchenko, "Proximitized materials," Mater. Today 22, 85 - 107 (2019)

[7] Gang Xu, Hongming Weng, Zhijun Wang, Xi Dai, and Zhong Fang, "Chern semimetal and the quantized anomalous Hall effect in $\mathrm{HgCr}_{2} \mathrm{Se}_{4}$," Phys. Rev. Lett. 107, 186806 (2011)

[8] T. Jungwirth, X. Marti, P. Wadley, and J. Wunderlich, "Antiferromagnetic spintronics," Nat. Nanotech. 11, 231-241 (2016).

[9] Hui Li, Shuangchen Ruan, and Yu-Jia Zeng, "Intrinsic van der Waals magnetic materials from bulk to the 2D limit: New frontiers of spintronics," Adv. Mater. 0, 1900065 (2019).

[10] L. Webster and Jia-An Yan, "Strain-tunable magnetic anisotropy in monolayer $\mathrm{CrCl}_{3}, \mathrm{CrBr}_{3}$, and $\mathrm{CrI}_{3}$," Phys. Rev. B 98, 144411 (2018)

[11] A. Haury, A. Wasiela, A. Arnoult, J. Cibert, S. Tatarenko, T. Dietl, and Y. Merle d'Aubigne, "Observation of a ferromagnetic transition induced by two-dimensional hole gas in modulation-doped CdMnTe quantum wells," Phys. Rev. Lett. 79, 511 (1997).

[12] B. Huang, G. Clark, E. Navarro-Moratalla, D. R. Klein, Ran Cheng, K. L. Seyler, Ding Zhong, E. Schmidgall, M. A. McGuire, D. H. Cobden, Wang Yao, Di Xiao, P. Jarillo-Herrero, and Xiaodong $\mathrm{Xu}$, "Intrinsic van der Waals magnetic materials from bulk to the 2D limit: New frontiers of spintronics," Nature 546, 270 (2019)

[13] R. R. Gałązka, "Semimagnetic semiconductors," in Proceedings 14 th International Conference on the Physics of Semiconductors, Edinburgh 1978, edited by B.L.H. Wilson (IoP, Bristol, 1978) p. 133.

[14] J. K. Furdyna and J. Kossut, Diluted Magnetic Semiconductors, Semiconductors and Semimetals, Vol. 25 (Academic Press, New York, 1988).

[15] T. Dietl, "(Diluted) Magnetic Semiconductors," in Handbook of Semiconductors, Vol. 3B, edited by S. Mahajan (North Holland, Amsterdam, 1994) p. 1251.

[16] J. Spałek, A. Lewicki, Z. Tarnawski, J. K. Furdyna, R. R. Gałązka, and Z. Obuszko, "Magnetic susceptibility of semimagnetic semiconductors: The hightemperature regime and the role of superexchange," Phys. Rev. B 33, 3407 (1986)

[17] R.R. Gałązka, "II-VI compounds - Polish perspective," phys. stat. sol. (b) 243, 759 (2006).

[18] J. C. Andresen, H. G. Katzgraber, V. Oganesyan, and M. Schechter, "Existence of a thermodynamic spin-glass phase in the zero-concentration limit of anisotropic dipolar systems," Phys. Rev. X 4, 041016 (2014).

[19] in Introduction to the Physics of Diluted Magnetic Semiconductors edited by J. A. Gaj and J. Kossut (Springer, Berlin, 2010).

[20] M. Król, R. Mirek, K. Lekenta, J.-G. Rousset, D. Stephan, M. Nawrocki, M. Matuszewski, J. Szczytko, W. Pacuski, and B. Piętka, "Spin polarized semimagnetic exciton-polariton condensate in magnetic field," Sci. Rep. 8, 6694 (2018). 
[21] C. Betthausen, P. Giudici, A. Iankilevitch, C. Preis, V. Kolkovsky, M. Wiater, G. Karczewski, B. A. Piot, J. Kunc, M. Potemski, T. Wojtowicz, and D. Weiss, "Fractional quantum Hall effect in a dilute magnetic semiconductor," Phys. Rev. B 90, 115302 (2014).

[22] R. Fiederling, M. Keim, G. Reuscher, W. Ossau, G. Schmidt, A. Waag, and L. W. Molenkamp, "Injection and detection of a spin-polarized current in a lightemitting diode," Nature 402, 787 (1999)

[23] B. Leclercq, C. Rigaux, A. Mycielski, and M. Menant, "Critical dynamics in $\mathrm{Cd}_{1-x} \mathrm{Mn}_{x} \mathrm{Te}$ spin glasses," Phys. Rev. B 47, 6169-6172 (1993).

[24] J. Jaroszyński, J. Wróbel, G. Karczewski, T. Wojtowicz, and T.Dietl, "Magnetoconductance noise and irreversibilities in submicron wires of spin-glass $\mathrm{n}^{+}$$\mathrm{Cd}_{1-x} \mathrm{Mn}_{x}$ Te," Phys. Rev. Lett. 80, 5635 (1998).

[25] T. Dietl, "Spin dynamics of a confined electron interacting with magnetic or nuclear spins: A semiclassical approach," Phys. Rev. B 91, 125204 (2015).

[26] T. Dietl, "Hole states in wide band-gap diluted magnetic semiconductors and oxides," Phys. Rev. B 77, 085208 (2008)

[27] W. Pacuski, P. Kossacki, D. Ferrand, A. Golnik, J. Cibert, M. Wegscheider, A. Navarro-Quezada, A. Bonanni, M. Kiecana, M. Sawicki, and T. Dietl, "Observation of strong-coupling effects in a diluted magnetic semiconductor $\mathrm{Ga}_{1-x} \mathrm{Fe}_{x} \mathrm{~N}$," Phys. Rev. Lett. 100, 037204 (2008).

[28] L. Besombes, Y. Léger, L. Maingault, D. Ferrand, H. Mariette, and $\mathrm{J}$. Cibert, "Probing the spin state of a single magnetic ion in an individual quantum dot," Phys. Rev. Lett. 93, 207403 (2004)

[29] J. Kobak, T. Smoleński, M. Goryca, M. Papaj, A. Bogucki K. Gietka, M. Koperski, J.-G. Rousset, J. Suffczyński, E. Janik, M. Nawrocki, A. Golnik, P. Kossacki, and W. Pacuski, "Designing quantum dots for solotronics," Nat. Commun. 5, 3191 (2014)

[30] T. Dietl, H. Ohno, F. Matsukura, J. Cibert, and D. Ferrand, "Zener model description of ferromagnetism in zinc-blende magnetic semiconductors," Science 287, 1019 (2000)

[31] T. Dietl and H. Ohno, "Dilute ferromagnetic semiconductors: Physics and spintronic structures," Rev. Mod. Phys. 86, 187-251 (2014).

[32] H. Ohno, "Making nonmagnetic semiconductors ferromagnetic," Science 281, 951 (1998).

[33] T. Story, R. R. Gałązka, R. B. Frankel, and P. A. Wolff, "Carrier-concentration-induced ferromagnetism in PbSnMnTe," Phys. Rev. Lett. 56, 777 (1986).

[34] D. Ferrand, J. Cibert, C. Bourgognon, S. Tatarenko, A. Wasiela, G. Fishman, A. Bonanni, H. Sitter, S. Koleśnik, J. Jaroszyński, A. Barcz, and T. Dietl, "Carrier-induced ferromagnetic interactions in p-doped $\mathrm{Zn}_{1-x} \mathrm{Mn}_{x} \mathrm{Te}$ epilayers," J. Cryst. Growth 214, 387 (2000)

[35] K. Olejník, M. H. S. Owen, V. Novák, J. Mašek, A. C. Irvine, J. Wunderlich, and T. Jungwirth, "Enhanced annealing, high Curie temperature and low-voltage gating in $(\mathrm{Ga}, \mathrm{Mn})$ As: A surface oxide control study," Phys. Rev. B 78, 054403 (2008)

[36] M. Wang, R. P. Campion, A. W. Rushforth, K. W. Edmonds, C. T. Foxon, and B. L. Gallagher, "Achieving high Curie temperature in (Ga,Mn)As," Appl. Phys. Lett. 93, 132103 (2008)
[37] Lin Chen, Xiang Yang, Fuhua Yang, Jianhua Zhao, J. Misuraca, Peng Xiong, and S. von Molnar̀, "Enhancing the Curie temperature of ferromagnetic semiconductor (Ga,Mn)As to $200 \mathrm{~K}$ via nanostructure engineering," Nano Lett. 11, 2584 (2011)

[38] Y. Fukuma, H. Asada, S. Miyawaki, T. Koyanagi, S. Senba, K. Goto, and H. Sato, "Carrierinduced ferromagnetism in $\mathrm{Ge}_{0.92} \mathrm{Mn}_{0.08} \mathrm{Te}$ epilayers with a Curie temperature up to $190 \mathrm{~K}$," Appl. Phys. Lett. 93, 252502 (2008).

[39] M. Hassan, G. Springholz, R.T. Lechner, H. Groiss, R. Kirchschlager, and G. Bauer, "Molecular beam epitaxy of single phase GeMnTe with high ferromagnetic transition temperature," J. Cryst. Growth 323, 363 (2011).

[40] Kan Zhao, Bijuan Chen, Guoqiang Zhao, Zhen Yuan, Qingqing Liu, Zheng Deng, Jinlong Zhu, and Changqing Jin, "Ferromagnetism at $230 \mathrm{~K}$ in $\left(\mathrm{Ba}_{0.7} \mathrm{~K}_{0.3}\right)\left(\mathrm{Zn}_{0.85} \mathrm{Mn}_{0.15}\right)_{2} \mathrm{As}_{2}$ diluted magnetic semiconductor," Chin. Sci. Bull. 59, 2524-2527 (2014).

[41] T. Andrearczyk, J. Jaroszyński, M. Sawicki, Le Van Khoi, T. Dietl, D. Ferrand, C. Bourgognon, J. Cibert, S. Tatarenko, T. Fukumura, Zhengwu Jin, H. Koinuma, and M. Kawasaki, "Ferromagnetic interactions in p- and n-type II-VI diluted magnetic semiconductors," in Proceedings 25th International Conference on Physics of Semiconductors, Osaka, Japan, 2000, edited by N. Miura and T. Ando (Springer, Berlin, 2001) p. 235.

[42] A. Kazakov, G. Simion, Y. Lyanda-Geller, V. Kolkovsky, Z. Adamus, G. Karczewski, T. Wojtowicz, and L. P. Rokhinson, "Mesoscopic transport in electrostatically defined spin-full channels in quantum Hall ferromagnets," Phys. Rev. Lett. 119, 046803 (2017)

[43] T. Jungwirth, J. Wunderlich, V. Novák, K. Olejník, B. L. Gallagher, R. P. Campion, K. W. Edmonds, A. W. Rushforth, A. J. Ferguson, and P. Němec, "Spin-dependent phenomena and device concepts explored in (Ga,Mn)As," Rev. Mod. Phys. 86, 855-896 (2014).

[44] Y. Ohno, D. K. Young, B. Beschoten, F. Matsukura, H. Ohno, and D. D. Awschalom, "Electrical spin injection in a ferromagnetic semiconductor heterostructure," Nature 402, 790 (1999)

[45] H. Ohno, D. Chiba, F. Matsukura, T. Omiya, E. Abe, T. Dietl, Y. Ohno, and K. Ohtani, "Electric-field control of ferromagnetism," Nature 408, 944 (2000).

[46] H. Boukari, P. Kossacki, M. Bertolini, D. Ferrand, J. Cibert, S. Tatarenko, A. Wasiela, J. A. Gaj, and T. Dietl, "Light and electric field control of ferromagnetism in magnetic quantum structures," Phys. Rev. Lett. 88, 207204 (2002).

[47] D. Chiba, M. Sawicki, Y. Nishitani, Y. Nakatani, F. Matsukura, and $\mathrm{H}$. Ohno, "Magnetization vector manipulation by electric fields," Nature 455, 515 (2008)

[48] M. Yamanouchi, D. Chiba, F. Matsukura, and H. Ohno, "Current-induced domain-wall switching in a ferromagnetic semiconductor structure," Nature 428, 539 (2004).

[49] M. Yamanouchi, D. Chiba, F. Matsukura, T. Dietl, and H. Ohno, "Velocity of domain-wall motion induced by electrical current in a ferromagnetic semiconductor (Ga,Mn)As," Phys. Rev. Lett. 96, 096601 (2006).

[50] C. Gould, C. Rüster, T. Jungwirth, E. Girgis, G. M. Schott, R. Giraud, K. Brunner, G. Schmidt, and L. W. Molenkamp, "Tunneling anisotropic magnetoresistance: A spin-valve like tunnel magnetoresistance using a single 
magnetic layer," Phys. Rev. Lett. 93, 117203 (2004).

[51] J. Wunderlich, T. Jungwirth, B. Kaestner, A. C. Irvine, A. B. Shick, N. Stone, K.-Y. Wang, U. Rana, A. D. Giddings, C. T. Foxon, R. P. Campion, D. A. Williams, and B. L. Gallagher, "Coulomb blockade anisotropic magnetoresistance effect in a (Ga,Mn)As single-electron transistor," Phys. Rev. Lett. 97, 077201 (2006).

[52] B. A. Bernevig and O. Vafek, "Piezomagnetoelectric effects in p-doped semiconductors," Phys. Rev. B 72, 033203 (2005)

[53] A. Chernyshov, M. Overby, Xinyu Liu, J. K. Furdyna, Y. Lyanda-Geller, and L. P. Rokhinson, "Evidence for reversible control of magnetization in a ferromagnetic material by means of spin-orbit magnetic field," Nat. Phys. 5, 656 (2009).

[54] S. Kanai, F. Matsukura, S. Ikeda, H. Sato, S. Fukami, and H. Ohno, "Spintronics: from basic research to VLSI application," AAPPS Bulletin 25, 4-11 (2015)

[55] T. Jungwirth, Q. Niu, and A. H. MacDonald, "Anomalous Hall effect in ferromagnetic semiconductors," Phys. Rev. Lett. 88, 207208 (2002).

[56] N. Nagaosa, J. Sinova, S. Onoda, A. H. MacDonald, and N. P. Ong, "Anomalous Hall effect," Rev. Mod. Phys. 82, 1539 (2010)

[57] He Ke, Yayu Wang, and Qi-Kun Xue, "Topological materials: quantum anomalous Hall system," Annu. Rev. Cond. Mat. Phys. 9, 329-349 (2018)

[58] Y. Tokura, K. Yasuda, and A. Tsukazaki, "Magnetic topological insulators," Nat. Rev. Phys. (2019), 110.1038/s42254-018-0011-5.

[59] A. Manchon, J. Zelezné, I. M. Miron, T. Jungwirth, J. Sinova, A. Thiaville, K. Garello, and P. Gambardella, "Current-induced spin-orbit torques in ferromagnetic and antiferromagnetic systems," Rev. Mod. Phys. (2019), in press, arXiv:1801.09636.

[60] S. Stefanowicz, G. Kunert, C. Simserides, J. A. Majewski, W. Stefanowicz, C. Kruse, S. Figge, Tian Li, R. Jakieła, K. N. Trohidou, A. Bonanni, D. Hommel, M. Sawicki, and T. Dietl, "Phase diagram and critical behavior of a random ferromagnet $\mathrm{Ga}_{1-x} \mathrm{Mn}_{x} \mathrm{~N}$," Phys. Rev. B 88, 081201(R) (2013).

[61] A. Bonanni, M. Sawicki, T. Devillers, W. Stefanowicz, B. Faina, Tian Li, T. E. Winkler, D. Sztenkiel, A. Navarro-Quezada, M. Rovezzi, R. Jakieła, A. Grois, M. Wegscheider, W. Jantsch, J. Suffczyński, F. D'Acapito, A. Meingast, G. Kothleitner, and T. Dietl, "Experimental probing of exchange interactions between localized spins in the dilute magnetic insulator (Ga,Mn)N," Phys. Rev. B 84, 035206 (2011).

[62] G. Kunert, S. Dobkowska, Tian Li, H. Reuther, C. Kruse, S. Figge, R. Jakiela, A. Bonanni, J. Grenzer, W. Stefanowicz, J. von Borany, M. Sawicki, T. Dietl, and D. Hommel, "Ga $\mathrm{Ga}_{1-x} \mathrm{mn}_{x} \mathrm{n}$ epitaxial films with high magnetization," Appl. Phys. Lett. 101, 022413-1 - 022413-4 (2012)

[63] I. Ya. Korenblit, E. F. Shender, and B. I. Shklovsky, "Percolation approach to the phase transition in the very dilute ferromagnetic alloys," Phys. Lett. A 46, 275 (1973).

[64] D. Sztenkiel, M. Foltyn, G.P. Mazur, R. Adhikari, K. Kosiel, K. Gas, R. Kruszka M. Zgirski, R. Jakiela, Tian Li, A. Piotrowska, A. Bonanni, M. Sawicki, and T. Dietl, "Stretching magnetism with an electric field in a nitride semiconductor," Nat. Commun. 7, 13232 (2016).
[65] Cui-Zu Chang, Jinsong Zhang, Xiao Feng, Jie Shen, Zuocheng Zhang, Minghua Guo, Kang Li, Yunbo Ou, Pang Wei, Li-Li Wang, Zhong-Qing Ji, Yang Feng, Shuaihua Ji, Xi Chen, Jinfeng Jia, Xi Dai, Zhong Fang, ShouCheng Zhang, Ke He, Yayu Wang, Li Lu, Xu-Cun Ma, and Qi-Kun Xue, "Experimental observation of the quantum anomalous Hall effect in a magnetic topological insulator," Science 340, 167-170 (2013).

[66] Rui Yu, Wei Zhang, Hai-Jun Zhang, Shou-Cheng Zhang, Xi Dai, and Zhong Fang, "Quantized anomalous Hall effect in magnetic topological insulators," Science 329, 61-64 (2010)

[67] Yabin Fan, Xufeng Kou, Pramey Upadhyaya, Qiming Shao, Lei Pan, Murong Lang, Xiaoyu Che, Jianshi Tang, Mohammad Montazeri, Koichi Murata, et al., "Electric-field control of spin-orbit torque in a magnetically doped topological insulator," Nature Nanotechnol. , 352 (2016).

[68] D. Bulmash, Chao-Xing Liu, and Xiao-Liang Qi, "Prediction of a Weyl semimetal in $\mathrm{Hg}_{1-x-y} \mathrm{Cd}_{x} \mathrm{Mn}_{y} \mathrm{Te}$," Phys. Rev. B 89, 081106 (2014).

[69] T. Dietl, H. Ohno, and F. Matsukura, "Hole-mediated ferromagnetism in tetrahedrally coordinated semiconductors," Phys. Rev. B 63, 195205 (2001)

[70] C. Lewinert and G. Bastard, "Indirect exchange interaction in extremely non-parabolic zero-gap semiconductors," J. Phys. C 13, 2347-60 (1980)

[71] M. G. Vergniory, M. M. Otrokov, D. Thonig, M. Hoffmann, I. V. Maznichenko, M. Geilhufe, X. Zubizarreta, S. Ostanin, A. Marmodoro, J. Henk, W. Hergert, I. Mertig, E. V. Chulkov, and A. Ernst, "Exchange interaction and its tuning in magnetic binary chalcogenides," Phys. Rev. B 89, 165202 (2014).

[72] S. Gupta, S. Kanai, F. Matsukura, and H. Ohno, "Magnetic and transport properties of $\mathrm{Sb}_{2} \mathrm{Te}_{3}$ doped with high concentration of Cr," Appl. Phys. Express 10, 103001 (2017).

[73] E. O. Lachman, A. F. Young, A. Richardella, J. Cuppens, H. R. Naren, Y. Anahory, A. Y. Meltzer, A. Kandala, S. Kempinger, Y. Myasoedov, M. E. Huber, N. Samarth, and E. Zeldov, "Visualization of superparamagnetic dynamics in magnetic topological insulators," Sci. Adv. 1 (2015), 10.1126/sciadv.1500740

[74] M. Götz, K. M. Fijalkowski, E. Pesel, M. Hartl, S. Schreyeck, M. Winnerlein, S. Grauer, H. Scherer, K. Brunner, C. Gould, F. J. Ahlers, and L. W. Molenkamp, "Precision measurement of the quantized anomalous Hall resistance at zero magnetic field," Appl. Phys. Lett. 112, 072102 (2018)

[75] E. J. Fox, I. T. Rosen, Yanfei Yang, G.e R. Jones, R. E. Elmquist, Xufeng Kou, Lei Pan, Kang L. Wang, and D. Goldhaber-Gordon, "Part-per-million quantization and current-induced breakdown of the quantum anomalous Hall effect," Phys. Rev. B 98, 075145 (2018)

[76] M. Pohlit, S. Rößler, Y. Ohno, S. Ohno, H.and von Molnár, Z. Fisk, J. Müller, and S. Wirth, "Evidence for ferromagnetic clusters in the colossal-magnetoresistance material $\mathrm{EuB}_{6}$," Phys. Rev. Lett. 120, 257201 (2018)

[77] T. Dietl, "Interplay between carrier localization and magnetism in diluted magnetic and ferromagnetic semiconductors," J. Phys. Soc. Jpn. 77, 031005 (2008)

[78] M. Sawicki, D. Chiba, A. Korbecka, Yu Nishitani, J. A. Majewski, F. Matsukura, T. Dietl, and 
H. Ohno, "Experimental probing of the interplay between ferromagnetism and localization in (Ga, Mn)As," Nat. Phys. 6, 22 (2010)

[79] A. Richardella, P. Roushan, S. Mack, B. Zhou, D. A. Huse, D. D. Awschalom, and A. Yazdani, "Visualizing critical correlations near the metal-insulator transition in $\mathrm{Ga}_{1-x} \mathrm{Mn}_{x}$ As," Science 327, 665 (2010).

[80] S. Kuroda, N. Nishizawa, K. Takita, M. Mitome, Y. Bando, K. Osuch, and T. Dietl, "Origin and control of high temperature ferromagnetism in semiconductors," Nat. Mater. 6, 440 (2007)

[81] A. Bonanni, A. Navarro-Quezada, T. Li, M. Wegscheider, Z. Matĕj, V. Holý, R. T. Lechner, G. Bauer, M. Rovezzi, F. D'Acapito, M. Kiecana, M. Sawicki, and T. Dietl, "Controlled aggregation of magnetic ions in a semiconductor: An experimental demonstration," Phys. Rev. Lett. 101, 135502 (2008).

[82] A. Bonanni, "(Nano)characterization of semiconductor materials and structures," Semicon. Sci. Technol. 26, 060301 (2011).

[83] T. Dietl, K. Sato, T. Fukushima, A. Bonanni, M. Jamet, A. Barski, S. Kuroda, M. Tanaka, Pham Nam Hai, and H. Katayama-Yoshida, "Spinodal nanodecomposition in semiconductors doped with transition metals," Rev. Mod. Phys. 87, 1311-1377 (2015).

[84] K. Sato, L. Bergqvist, J. Kudrnovský, P. H. Dederichs, O. Eriksson, I. Turek, B. Sanyal, G. Bouzerar, H. Katayama-Yoshida, V. A. Dinh, T. Fukushima, H. Kizaki, and R. Zeller, "Firstprinciples theory of dilute magnetic semiconductors," Rev. Mod. Phys. 82, 1633 (2010)

[85] A. Bonanni and T. Dietl, "A story of hightemperature ferromagnetism in semiconductors," Rev. Chem. Soc. 39, 528 (2009).

[86] M. Birowska, C. Śliwa, J. A. Majewski, and T. Dietl, "Origin of bulk uniaxial anisotropy in zinc-blende dilute magnetic semiconductors," Phys. Rev. Lett. 108, 237203 (2012)

[87] Ye Yuan, R. Hübner, M. Birowska, Chi Xu, Mao Wang, S. Prucnal, R. Jakiela, K. Potzger, R. Böttger, S. Facsko, J. A. Majewski, M. Helm, M. Sawicki, Shengqiang Zhou, and T. Dietl, "Nematicity of correlated systems driven by anisotropic chemical phase separation," Phys. Rev. Materials 2, 114601 (2018). 